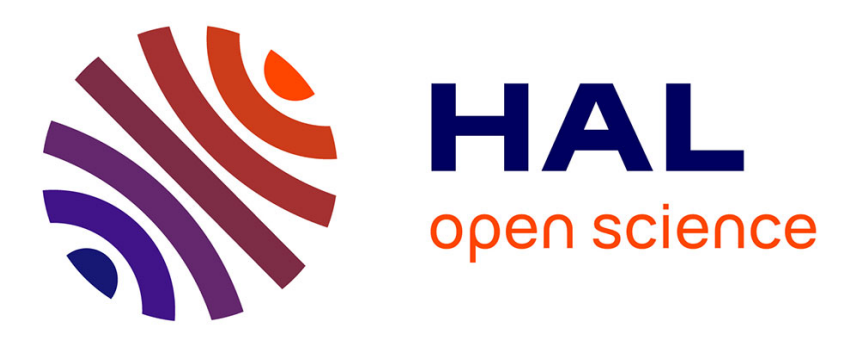

\title{
Une commune résidentielle confrontée au problème de l'aménagement de son territoire: Le cas de Gargas (Lubéron)
}

Cécile Helle, Anne-Elisabeth Laques

\section{- To cite this version:}

Cécile Helle, Anne-Elisabeth Laques. Une commune résidentielle confrontée au problème de l'aménagement de son territoire: Le cas de Gargas (Lubéron). L'Information géographique, 1998, 62 (1), pp.28 - 33. 10.3406/ingeo.1998.2568 . hal-01837072

\section{HAL Id: hal-01837072 \\ https://hal.science/hal-01837072}

Submitted on 12 Jul 2018

HAL is a multi-disciplinary open access archive for the deposit and dissemination of scientific research documents, whether they are published or not. The documents may come from teaching and research institutions in France or abroad, or from public or private research centers.
L'archive ouverte pluridisciplinaire HAL, est destinée au dépôt et à la diffusion de documents scientifiques de niveau recherche, publiés ou non, émanant des établissements d'enseignement et de recherche français ou étrangers, des laboratoires publics ou privés. 


\section{Une commune résidentielle confrontée au problème de} l'aménagement de son territoire : Le cas de Gargas (Lubéron)

Cécile Helle, Anne-Elisabeth Laques

\section{Citer ce document / Cite this document :}

Helle Cécile, Laques Anne-Elisabeth. Une commune résidentielle confrontée au problème de l'aménagement de son territoire : Le cas de Gargas (Lubéron). In: L'information géographique, volume 62, n¹, 1998. pp. 28-33;

doi : https://doi.org/10.3406/ingeo.1998.2568

https://www.persee.fr/doc/ingeo_0020-0093_1998_num_62_1_2568

Fichier pdf généré le 09/05/2018 


\title{
Une commune résidentielle confrontée au problème de l'aménagement de son territoire Le cas de Gargas (Lubéron)
}

\author{
Cécile Helle \\ Laboratoire d'Analyse Spatiale. \\ UMR Espace (Nice)
}

Anne-Elisabeth Laques

Laboratoire Structures et Dynamiques Spatiales. UMR Espace (Avignon)

L 'urbanisation est aujourd'hui l'un des processus majeurs de structuration et de dynamique des territoires. Sous son impulsion, de nouvelles solidarités s'établissent entre villes et périphéries, des dichotomies de plus en plus marquées s'observent entre cellule de résidence et lieu de travail, des paysages hybrides où se mêlent caractères agricoles et urbains progressent. Surtout se créent et se diffusent des objets matériels inscrits durablement dans l'espace, fabriquant et modelant de nouveaux territoires, établissant et imposant de nouvelles formes d'organisation spatiale. L'inscription physique de la ville dans son environnement, par l'avancée des sols bâtis, demeure de fait le caractère le plus visible de ce phénomène et constitue l'un des principaux moteurs de la formation et de la transformation des espaces produits et vécus par les sociétés humaines. Les zones résidentielles les plus récemment édifiées apparaissent dès lors comme des champs d'exploration privilégiés pour observer les mécanismes de production, de réaffectation, de consommation et de spécialisation spatiales induits par cette dynamique.

Sur la commune de Gargas (dont peu d'éléments rappellent aujourd'hui qu'elle est située dans l'un des hauts lieux de villégiature d'Europe, le Lubéron. (fig $\mathrm{n}^{\circ} 1$ ), le front de colonisation du bâti a évolué très rapidement et très intensément depuis deux décennies aux dépens de l'ancien terroir agricole. Devenu un simple quartier périphérique de la petite ville limitrophe d'Apt, sa popula- tion a été multipliée par trois entre 1975 et 1990 (progressant de 1064 à 2875 habitants) et sa dépendance en termes d'emploi est passée au cours de la même période de 38 à $50 \%$. Mais audelà de cette solidarité fonctionnelle et de la remise en cause des équilibres communautaires, c'est la coalescence morphologique établie entre ville-centre et commune périphérique qui a été la plus marquante. La forte progression de quartiers pavillonnaires juxtaposés sans réels liens entre eux et l'imbrication de zones résidentielles, industrielles et commerciales ont profondément modifié l'organisation spatiale de cette localité et ont participé à une certaine banalisation des paysages agricoles traditionnels des régions méditerranéennes.

\section{Figure 1 : localisation de Gargas}

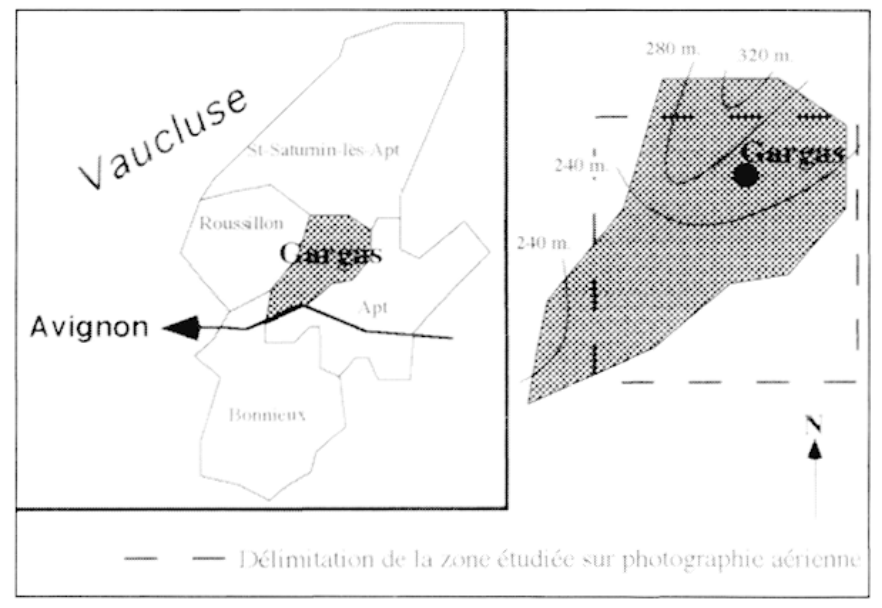


Pour localiser et mesurer ces formes de diffusion résidentielle et pour reconnaître leurs incidences, un Système d'Information Géographique en mode raster est mis au point. Ses plans d'information sont élaborés à partir d'un travail de photo-interprétation sur photographies aériennes (note méthodologique). Outre l'identité de structuration ainsi établie entre outil et information (monde de l'image et du pixel), cette approche coïncide bien avec l'orientation résolument spa- tiale et dynamique adoptée. Mais les S.I.G. sont avant tout descriptifs; aussi la compréhension des processus et des logiques d'acteurs à l'origine de ces nouveaux territoires de vie quotidienne contraint-elle à mobiliser d'autres documents, en l'occurrence les Plans d'Occupation des Sols réalisés sous la responsabilité des municipalités depuis les lois de Décentralisation et qui fixent les conditions d'extension du bâti dans l'infracommunal.

Figure 2 : surfaces bâties en 1969, 1984 et 1991

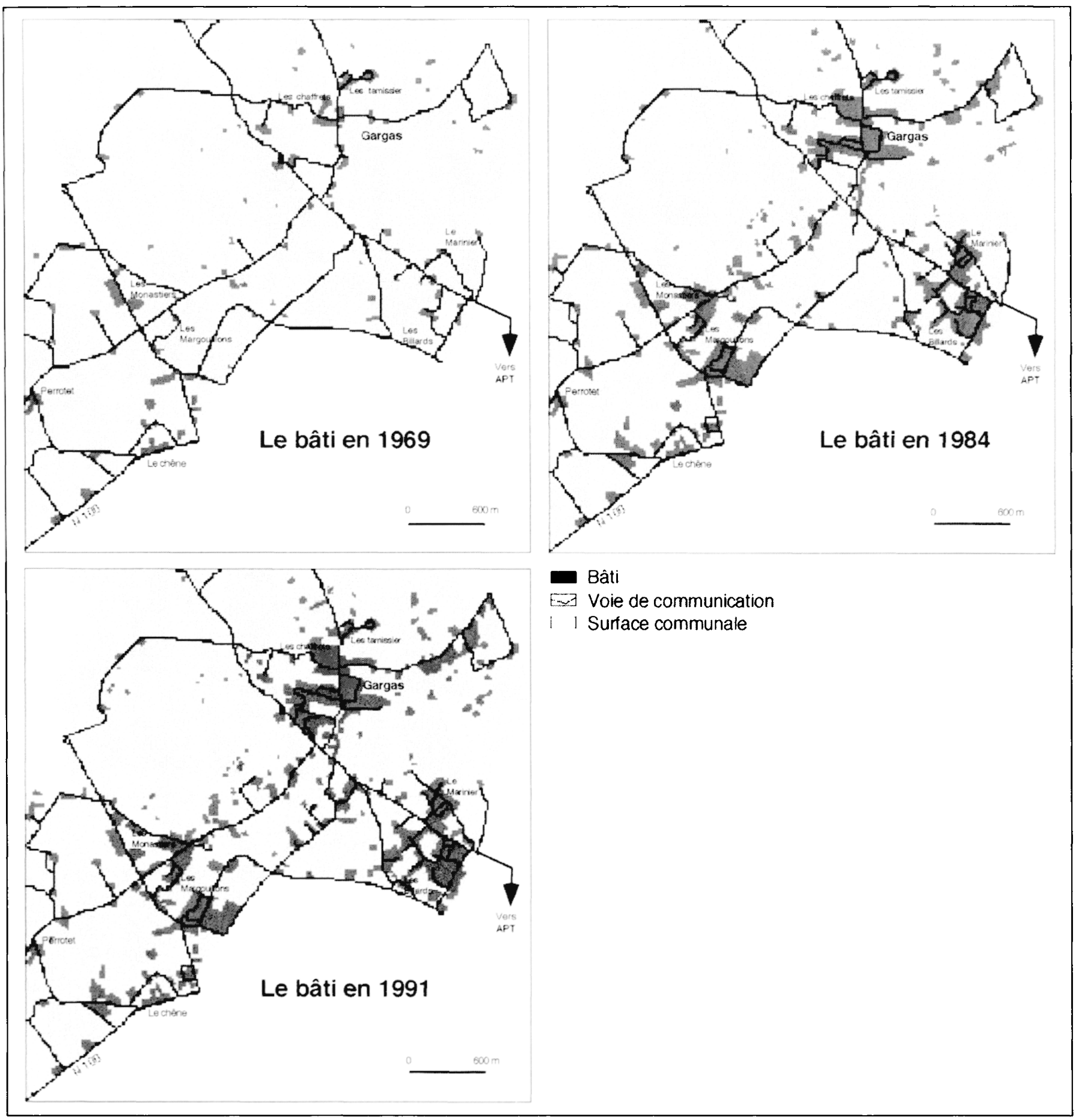




\section{LA DIFFUSION DU BÂTI SUR LA COMMUNE DE GARGAS}

Les cartes des surfaces bâties en 1969, 1984 et 1991 (fig. 2) apportent un premier niveau d'information sur l'importante densification résidentielle qui affecte la commune de Gargas depuis près de vingt ans. Seule la partie nord-ouest, la plus éloignée de la ville d'Apt et la plus boisée, est restée étrangère à toute diffusion des constructions. Partout ailleurs, celle-ci a par contre été intensive, surtout au cours de la première période : de 1969 à 1984, les surfaces bâties ont progressé de $268 \%$ contre $23 \%$ de 1984 à 1991 (fig. 3). Généralisée et dispersée sur l'ensemble du territoire, cette dynamique résidentielle ne procède pas d'une logique auréolaire classique : aucune diminution des densités de constructions avec l'augmentation de la distance au noyau villageois n'est réellement décelée. En effet la commune de Gargas ne dispose pas de

Figure 3 : la diffusion du bâti de 1969 à 1991

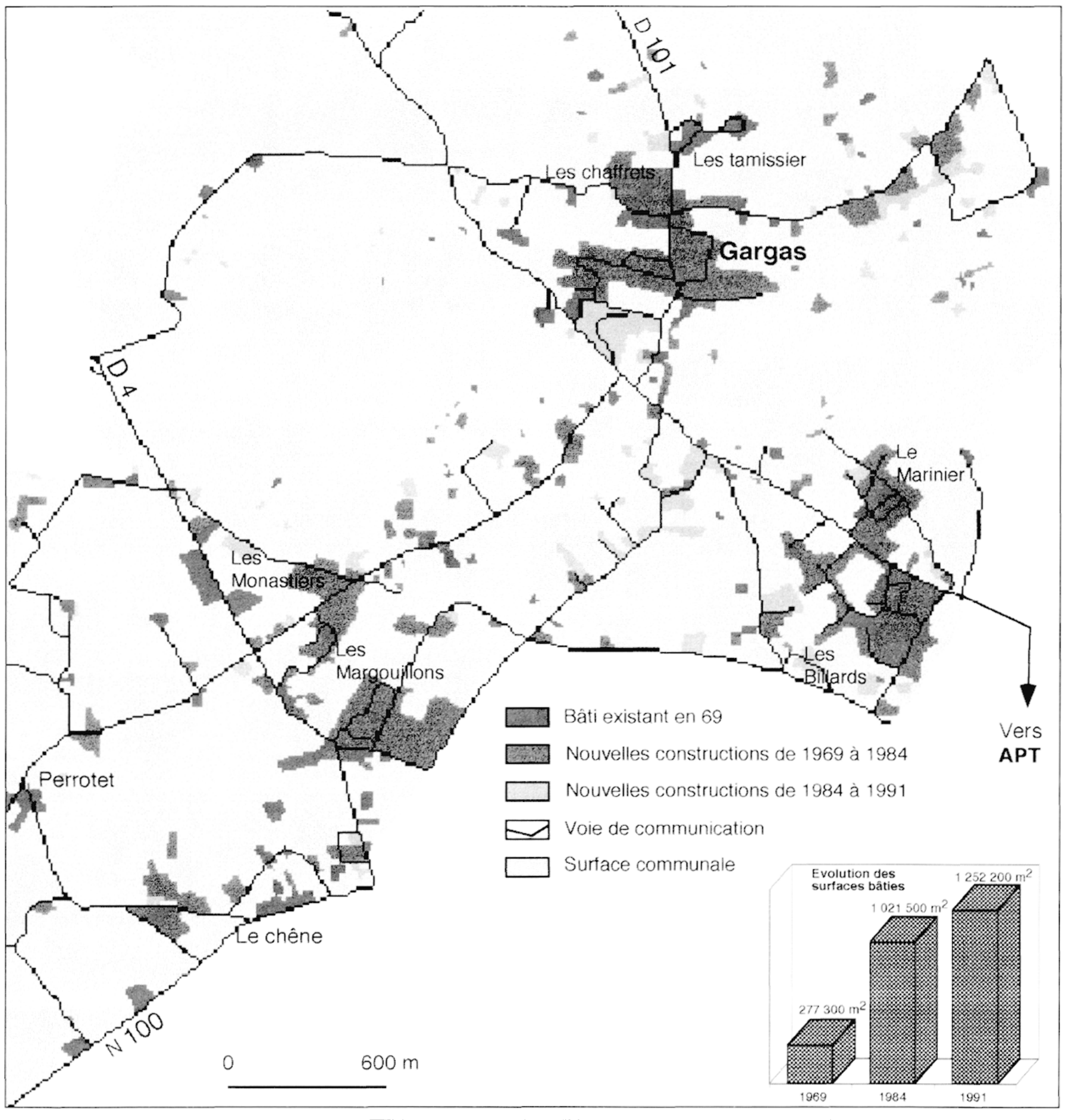


véritable centre ancien; en outre les quelques hameaux qui ont présidé à sa constitution n'ont que peu influencé le processus de diffusion. Ces éléments et la localisation périphérique de deux des quartiers nouvellement bâtis (quartiers les Billards et les Margouillons) corroborent l'idée d'une urbanisation guidée par l'accessibilité à la ville-centre, Apt. C'est en effet dans la zone frontalière et le long d'axes majeurs de communication (départementales 101 et 201 , branches secondaires de la nationale 100 , principale artère de la ville d'Apt) que se sont produites la plupart des concentrations résidentielles.

Enfin cette urbanisation s'est préférentiellement réalisée sous forme de lotissements caractérisés par une partition territoriale relativement fine (parcelles constructibles de 800 à 1200 mètres carré) et par un treillage régulier et rectiligne des voies de desserte, aboutissant au maillage en damier des

Figure 4 : les types de cultures évincés par le bâti

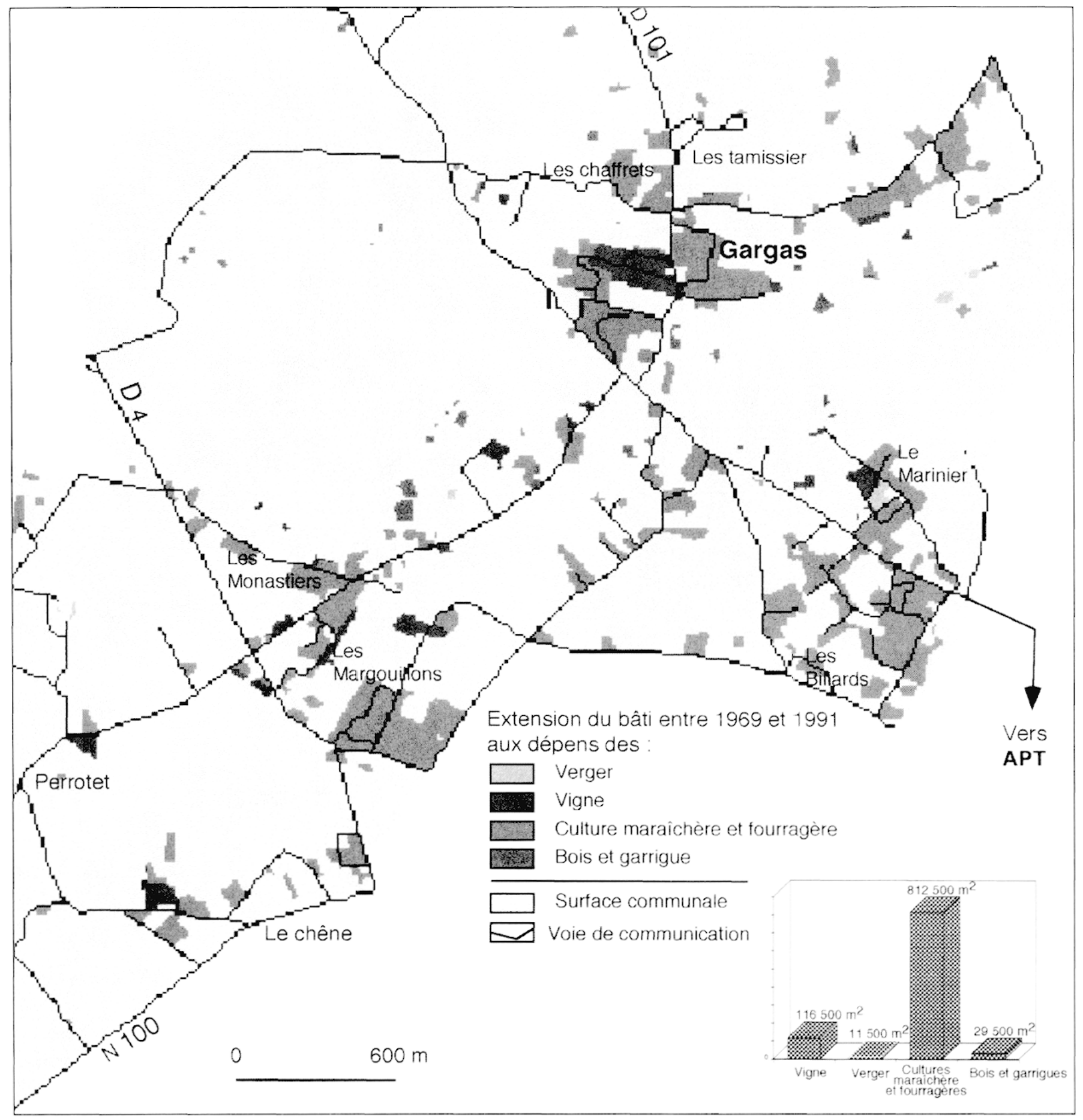


nouveaux espaces bâtis. Elle a été orchestrée par les professionnels de l'immobilier qui dominent plus du quart du marché des terrains à bâtir et qui réalisent la quasi totalité des opérations groupées d'habitat individuel.

\section{CONSOMMATION SPATIALE ET ESPACE COLONISE}

Grâce à l'une des opérations spatiales les plus classiques des Systèmes d'Information Géographique - l'opération de combinaison -, il est possible de qualifier et quantifier les processus de réaffectation induits par la dynamique urbaine.

A la fin des années soixante-dix, le terroir de la commune de Gargas se caractérise principalement par l'étendue importante des surfaces cultivées et par le cantonnement des secteurs bâtis aux hameaux et à leur périphérie proche. L'association des cultures maraîchères, fruitières et viticoles compose alors des paysages agricoles fortement identitaires de la Provence intérieure. S'y ajoutent de vastes pinèdes développées sur les sols siliceux des anciennes carrières d'ocre.

Vingt ans plus tard, la diffusion progressive du bâti individuel, fort consommateur d'espace, a engendré simultanément la contraction et le morcellement des terres cultivées. Des résistances différenciées peuvent néanmoins être décelées en fonction des types de cultures initialement pratiquées (fig. 4). Les terres maraîchères ont été les premières à pâtir de l'avancée du monde urbain, tandis que les vergers comme le vignoble ont moins souffert de ce processus de conquête territoriale. D'autres zones méditerranéennes (Comtat, région marseillaise) présentent des dynamiques agricoles identiques : les cultures légumières ne parviennent à se maintenir face à la logique résidentielle du monde urbain que par le développement des productions sous serres. Dans le Pays d'Apt, la résistance des vergers et vignes s'explique par le relatif maintien de la rentabilité de la culture de la cerise destinée à la confiserie (Apt, capitale mondiale des fruits confits) et par la bonne commercialisation des côtes-du-Luberon reconnus depuis quelques années par une Appellation d'Origine Contrôlée. En outre loin d'être une simple boisson, ces bouteilles de vin offrent à tout acheteur une part de rêve - la réminiscence d'une luminosité exceptionnelle ou le souvenir d'une richesse culturelle -.

\section{DYNAMIQUE RÉSIDENTIELLE ET POLITIQUE D'URBANISME}

L'intense diffusion du bâti observée sur la commune de Gargas depuis vingt ans reflète les choix urbanistiques retenus par les municipalités successives. De 1977 à 1991, le plan d'occupation des sols en vigueur prône le tout-urbain et le toujours plus de constructions, sans prendre convenablement la mesure des incidences de ce processus sur le devenir de la localité (fig. 5).

Plus de $60 \%$ du territoire communal se trouve alors potentiellement ouvert à la construction. Les terres cultivées sont certes classées en zone inconstructible, mais les agriculteurs, leurs descendants et ascendants directs, enfin les propriétaires de terrains déclarés incultes par le Conseil Municipal sont autorisés à y édifier des bâtiments à des fins d'habitat. De même dans les espaces boisés, seul le secteur des carrières d'ocre se trouve totalement protégé de toute propagation du bâti ; ailleurs celleci est autorisée sous réserve toutefois d'acquérir des parcelles de plus de $10000 \mathrm{~m}^{2}$.

\section{Note méthodologique}

Un seul type de documents a servi de source d'information pour procéder à l'analyse du processus de diffusion du bâti sur la commune de Gargas. Il s'agit des photographies aériennes des missions de 1969, 1984 et 1991. Ces dates correspondent aux seules missions effectuées par l'I.G.N. au cours des vingt dernières années. Elles ont comme avantage de coïncider avec les dates des recensements de population de l'INSEE (respectivement 1968, 1982 et 1990) et comme inconvénient de définir des plages de termps inégales, quatorze ans pour la première période et huit ans pour la seconde.

Ces données spatialisées ont été prétraitées dans le logiciel de retouche d'images Adobe Photoshop pour aboutir à l'élaboration de ce qui constitue réellement les données de base du Système d'Information Géographique : les plans d'information sur l'occupation du sol. Cette phase de prétraitement, très fastidieuse et longue, comprend outre la photo-interprétation des clichés aériens, des opérations de réajustement et de redressement (ce travail a été réalisé par P. Ellerkamp, actuellement allocataire-moniteur du laboratoire Structures et Dynamiques Spatiales).

Une fois construites, ces informations spatialisées ont été exportées sous le logiciel Map II (S.I.G. raster). I es opérations d'extraction (Recode) et de combinaison (Combine) qui sont parmi les plus usuelles des Systèmes d'Information Géographique ont été mobilisées pour percevoir, qualifier et quantifier les dynamiques urbaines de la commune de Gargas. 
Ce n'est qu'à partir de 1989 avec l'élection d'une nouvelle équipe municipale qu'émerge une autre politique fondée sur la maîtrise de l'urbanisation. Pour répondre à cet objectif, la procédure de révision du Plan d'Occupation des Sols aboutit au reclassement de terrains à bâtir en terres agricoles, à l'instauration de seuils minimum élevés pour pouvoir bâtir dans les zones d'urbanisation diffuse, enfin à la définition de réserves foncières (fig. 5).

Depuis 1969, la densification résidentielle s'est produite avec une telle intensité sur la commune de Gargas qu'elle a entraîné la déstructuration du terroir agricole. Compartimenté par des îlots résidentiels en constante expansion, ce dernier a perdu de son homogénéité tant d'un point de vue fonctionnel que d'un point de vue paysager. L'imbrication des fonctions résidentielles et agricoles, voire industrielles et commerciales augmente en effet la confusion dans l'affectation des lieux, mi-urbain, mi-rural ; elle participe aussi à la standardisation des paysages du péri-urbain.

Face à cette situation et en dépit de l'aide apportée par le Parc Naturel Régional du Luberon, la municipalité se trouve bien démunie. Son action se cantonne nécessairement à la gestion de l'existant : on a encore rarement vu des bulldozers raser des lotissements. Toutefois le contrôle de la diffusion du bâti s'insère dans un projet plus global d'aménagement du territoire dont les deux autres volets sont la protection des sites et paysages associés à l'exploitation de l'ocre et la création d'un véritable noyau villageois par l'édification de nouveaux équipements publics et de quelques logements sociaux greffés sur la concentration résidentielle en position la plus centrale dans la commune (quartiers les Janselmes et les Sauvans). Le but ultime est de tenter de doter Gargas d'une réelle identité et d'y insuffler une vraie vie communautaire.

Figure 5 : les plans d'occupation des sols de la commune de Gargas

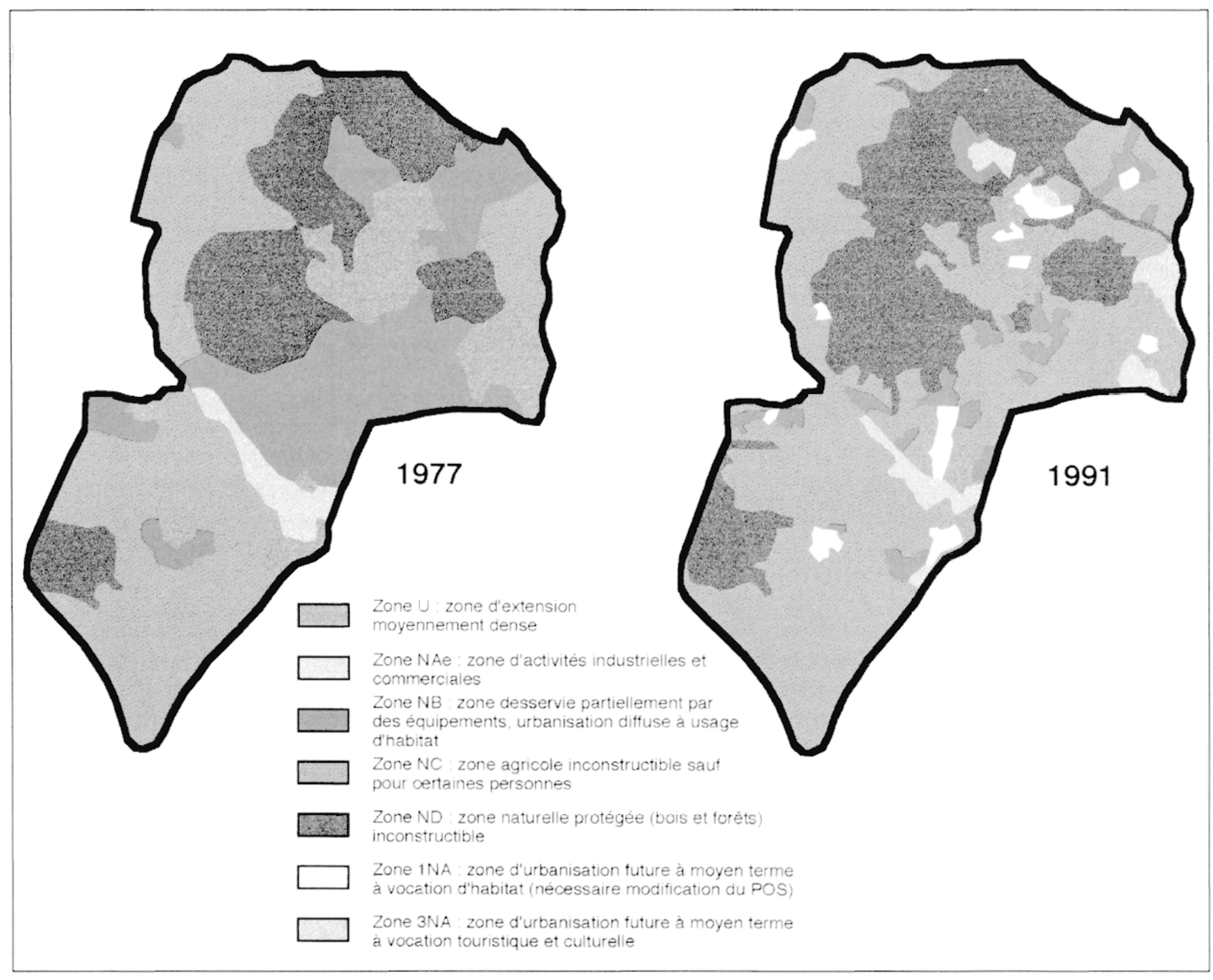

